\title{
Resonant Modes of a Waveguide Iris Discontinuity: Interpretation in Terms of Canonical Circuits
}

\author{
Raúl Rodríguez-Berral ${ }^{\circledR}$, Francisco Mesa ${ }^{\circledR}$, Fellow, IEEE, and Francisco Medina ${ }^{\circledR}$, Fellow, IEEE
}

\begin{abstract}
This paper presents an eigenproblem for computing the resonances of multiresonant irislike discontinuities in waveguides. The formulation of the eigenproblem is based on integral equation methods, and its solution provides the resonance frequencies (eigenvalues) and the associated resonant aperture field's patterns (eigenvectors). These eigenvectors can be used as a basis for the expansion of the aperture fields at any frequency within the band of interest, thus leading to a significant reduction of the size of the method-of-moments admittance matrix. Moreover, under certain approximations, the orthogonality of the eigenvectors allows for the derivation of a Foster-like canonical equivalent circuit with capacitances and inductances that present a smooth frequency dependence. In order to obtain an equivalent Foster circuit with frequencyindependent inductors and capacitors, a straightforward and simple iterative refinement is proposed. The circuit thus obtained accurately reproduces the response of the multiresonant irislike discontinuity over a wide frequency band.
\end{abstract}

Index Terms-Circuit models, Foster's synthesis, integral equation (IE), waveguide discontinuities.

\section{INTRODUCTION}

$\mathbf{T}$ HE presence of metallic discontinuities (iris/obstacle) inside the waveguides has been used since mid-1900s to create localized regions of electric/magnetic stored energy that allows for the control of the scattering parameters of the fundamental propagating mode [1]-[7]. Appropriate models were reported to account for the effect of those discontinuities [8]-[10], and their application to the design of filters and other passive waveguide devices became a common practice. As is well known, the basic electromagnetic modeling of such discontinuities lies in transmission line theory and the characterization of the excess magnetic/electric energy as lumped inductors/capacitors in an equivalent network. The simplest case occurs when the discontinuity is planar and infinitely thin along the propagation direction, and the

Manuscript received August 4, 2017; revised November 30, 2017; accepted January 6, 2018. Date of publication February 28, 2018; date of current version May 4, 2018. This work was supported in part by the Spanish Ministerio de Economía y Competitividad with European Union FEDER Funds under Project TEC2013-41913-P and Project TEC2017-84724-P and in part by the Spanish Junta de Andalucía under Project P12-TIC-1435. (Corresponding author: Raúl Rodríguez-Berral.)

R. Rodríguez-Berral and F. Mesa are with the Microwaves Group, Departamento de Física Aplicada 1, ETS de Ingeniería Informática, Universidad de Sevilla, 41012 Seville, Spain (e-mail: rrberral@us.es; mesa@us.es).

F. Medina is with the Facultad de Física, Departamento de Electrónica y Electromagnetismo, Universidad de Sevilla, 41012 Seville, Spain (e-mail: medina@us.es).

Color versions of one or more of the figures in this paper are available online at http://ieeexplore.ieee.org.

Digital Object Identifier 10.1109/TMTT.2018.2804914 operation frequency is far from the cutoff frequency of the first higher order mode. In such a case, the discontinuity is well modeled by just a single $L C$ tank in parallel with two transmission lines accounting for the propagation of the fundamental mode at both sides of the discontinuity [8]-[10]. When the electrical size of the iris/obstacle scatterer approaches half the operating wavelength, resonances associated with this element start to appear, and the waveguide shows the expected passband/stopband behavior. If the discontinuity comprises multiple irises/patches, or a single iris/patch discontinuity shows more than one resonance at the frequency band of interest, a multiresonant discontinuity problem is faced. In this situation, Foster's reactance theorem ensures that such discontinuity can be synthesized by either shunttuned elements or series-tuned elements [8]. Although this theorem provides a valuable physical insight into the behavior of multiple-resonance discontinuities, it does not give any information on the values of the inductors and capacitors for a given discontinuity geometry. This information can easily be extracted from the knowledge of the poles and zeros of the discontinuity admittance, but certainly this knowledge is reached only after the electromagnetic problem has already been solved.

The microwave community has devoted a lot of effort to find approximate and/or rigorous solutions to this problem; in particular, for the case of irislike discontinuities, which are the object of study in this paper, some of the analysis methods reported in the literature can be found, for instance, in [11]-[24]. The initial motivation of the authors of this paper was to extend their previous efforts on the circuit modeling of the scattering of periodic structures [25]-[27] to find a wideband equivalent circuit model for a multiresonant irislike discontinuity. Since, according to the discussion ealier, the knowledge of the resonance frequencies provides valuable information in order to build up a Foster-like equivalent circuit, the authors' effort was focused on the development of a strategy to efficiently characterize the resonances of a multiresonant iris discontinuity inside a homogeneous waveguide. Within the framework of an integral equation (IE) formulation [14], [15], [28], [29], such a strategy is proposed here in the form of a novel generalized eigenvalue problem in which the eigenvalues provide the resonance frequencies and the eigenvectors are the corresponding resonant profiles of the aperture field (or the equivalent magnetic current at the apertures). As an interesting by-product, it is found that the resonant profiles extracted from the eigenproblem can 
be efficiently used as quasi-orthogonal basis functions that yield a convenient alternative IE-method-of-moments (MoM) scheme.

Here, it is worth noting that, at first sight, our procedure might seem to follow the same line of action as the so-called characteristic mode analysis (CMA), initially proposed in [30] and [31] for problems involving radiation and scattering by conducting objects and widely used thereafter [32]-[41]. However, despite the similarities in the mathematical formalism, it should be noted that the eigenproblem proposed in this paper is a different one, derived from enforcing the resonance condition and, in consequence, providing by construction a direct characterization of the resonances. Furthermore, to the best of our knowledge, the CMA is not suitable for application to discontinuities inside waveguides. Indeed, although it has been adapted and applied to different problems of radiating apertures [35]-[41], an extension of this procedure to the case of apertures inside waveguides does not seem to be feasible [42].

Back to our formulation, when the resonant profiles are used as basis functions in the MoM, their orthogonality properties lead to a simple interpretation of the resulting equation system in terms of an equivalent circuit with precisely the topology of Foster's first canonical form. In consequence, the present proposal straightforwardly leads to Foster's synthesis of the discontinuity, yielding a systematic semianalytic procedure to compute the values of the capacitors and inductors involved in the equivalent circuit. Although the eigenproblem proposed in this paper is actually frequencydependent, a relevant feature of our proposal is that this frequency dependence is found to be sufficiently smooth as to allow for an additional postprocessing that avoids the necessity of solving the eigenproblem for each frequency of interest (which is very advantageous when studying wideband operation).

This paper will be organized as follows. The basic formulation of the eigenproblem is presented in Section II. The discretization of the eigenproblem into matrix form is considered in Section III, which also shows numerical results that illustrate the usage of the resonant profiles as basis functions. The orthogonality properties of eigenvectors and the derivation of the Foster-like equivalent circuits for the problem are included in Section IV, together with some related numerical results, and Section V adds discussions about the excitation of the resonances and its interpretation within the frame of the obtained circuit model. Finally, Section VI shows additional results for a more complex discontinuity and introduces a further refinement strategy before drawing some conclusions in Section VII.

\section{Formulation of the Eigenproblem}

The general structure under analysis consists of a planar metallic iris discontinuity inside a homogeneous waveguide, which can present multiple apertures. An example with rectangular geometry and two apertures is shown in Fig. 1(a). Our initial goal is to obtain a formulation that characterizes the resonances of the discontinuity. A time-harmonic dependence of the type $\exp (\mathrm{j} \omega t)$ is assumed throughout this paper for

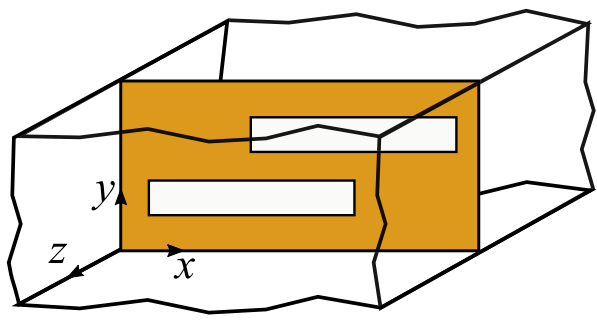

(a)

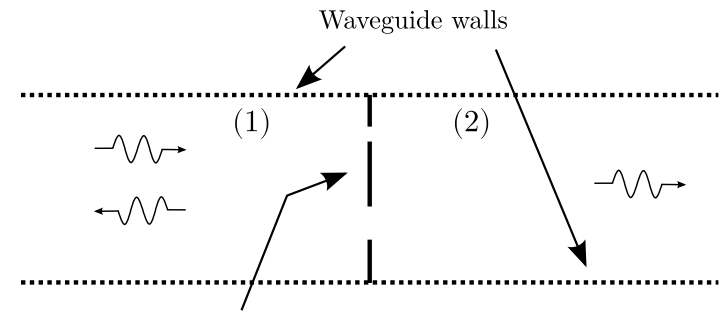

Planar metallic discontinuity

(b)

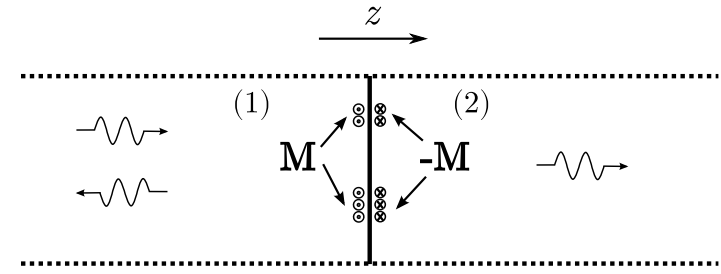

(c)

Fig. 1. (a) Example of the general structure under consideration. (b) Schematic lateral view. (c) Equivalent magnetic currents at the apertures.

all the field quantities, with $\omega$ being the angular frequency. In our procedure, and as usual in IE formulations, the apertures can be replaced with equivalent unknown magnetic currents placed at the aperture regions, as shown in Fig. 1(c). From the continuity of the magnetic field through the apertures, the IE for the unknown magnetic current $\mathbf{M}$ can be written in a compact form as

$$
2 \mathbf{H}_{\mathrm{inc}}-\hat{Y} \mathbf{M}=\mathbf{0}
$$

where $\mathbf{H}_{\text {inc }}$ is the magnetic field of the incident waveguide mode (in the absence of magnetic current, the incident mode is completely reflected, hence the factor 2) and $\hat{Y}$ is the admittance operator that yields the jump in the magnetic field due to $\mathbf{M}$; namely,

$$
\hat{Y} \mathbf{M}=\mathbf{H}_{2}[\mathbf{M}]_{z=0^{+}}-\mathbf{H}_{1}[\mathbf{M}]_{z=0^{-}}=2 \mathbf{H}_{2}[\mathbf{M}]_{z=0^{+}} .
$$

The admittance operator can easily be written as an expansion in terms of the waveguide modes. Thus, let $\mathbf{e}_{n}$ denote the transverse (to $z$ ) electric-field profile of the $n$th waveguide mode and $\mathbf{h}_{n}=\hat{\mathbf{z}} \times \mathbf{e}_{n}$ denote the corresponding magnetic field profile. These modal profiles are normalized so that

$$
\left\langle\mathbf{e}_{n}, \mathbf{e}_{m}\right\rangle=\left\langle\mathbf{h}_{n}, \mathbf{h}_{m}\right\rangle=\delta_{n m}
$$

where the following usual inner product notation is used for the projection integrals in the waveguide cross section $(S)$ :

$$
\langle\mathbf{f}, \mathbf{g}\rangle=\int_{S} \mathbf{f}^{*}(x, y) \cdot \mathbf{g}(x, y) \mathrm{d} S .
$$


Taking into account that the transverse electric field due to $\mathbf{M}$ at the discontinuity plane is the aperture field $\mathbf{E}_{\mathrm{a}}=\mathbf{M} \times \hat{\mathbf{z}}$, its modal expansion is clearly given by

$$
\left.\mathbf{E}\right|_{z=0}=\sum_{n}\left\langle\mathbf{e}_{n}, \mathbf{E}_{\mathbf{a}}\right\rangle \mathbf{e}_{n}=\sum_{n}\left\langle\mathbf{h}_{n}, \mathbf{M}\right\rangle \mathbf{e}_{n} .
$$

The expansion for the transverse magnetic field is then

$$
\left.\mathbf{H}_{2}\right|_{z=0^{+}}=-\left.\mathbf{H}_{1}\right|_{z=0^{-}}=\sum_{n} Y_{n}\left\langle\mathbf{h}_{n}, \mathbf{M}\right\rangle \mathbf{h}_{n}
$$

with $Y_{n}$ being the transverse wave admittance of the modes given by the ratio of the transverse modal field amplitudes, which can be written as

$$
Y_{n}=\frac{\left|\hat{\mathbf{z}} \times \mathbf{H}_{n}\right|}{\left|\hat{\mathbf{z}} \times \mathbf{E}_{n}\right|}= \begin{cases}\omega \varepsilon_{0} / \beta_{n}, & \text { TM modes } \\ \beta_{n} / \omega \mu_{0}, & \text { TE modes }\end{cases}
$$

where $\beta_{n}$ is the propagation wavenumber of the $n$th mode. In a lossless scenario, this wavenumber is real for propagative modes and imaginary for evanescent ones $\left(\beta_{n} \equiv-\mathrm{j} \alpha_{n}\right)$. From (2) and (6), it is apparent that

$$
\hat{Y} \mathbf{M}=\sum_{n} 2 Y_{n}\left\langle\mathbf{h}_{n}, \mathbf{M}\right\rangle \mathbf{h}_{n}=\sum_{n} 2 Y_{n} \hat{p}_{n} \mathbf{M}
$$

where

$$
\hat{p}_{n} \mathbf{M}=\left\langle\mathbf{h}_{n}, \mathbf{M}\right\rangle \mathbf{h}_{n}
$$

can be identified as the projection of $\mathbf{M}$ onto the $n$th mode magnetic field profile and $\hat{p}_{n}$ as the associated projection operator. In the absence of losses, the wave admittances $Y_{n}$ in (7) are real/imaginary for propagative/evanescent modes. In consequence, the $\hat{Y}$ operator is not Hermitian in general. It is then convenient to consider propagative and evanescent modes separately in the following way:

$$
\begin{aligned}
\hat{Y} \mathbf{M} & =\sum_{n \text { prop. }} 2 Y_{n} \hat{p}_{n} \mathbf{M}+\mathrm{j} \sum_{n \text { evan. }} 2 B_{n} \hat{p}_{n} \mathbf{M} \\
& =\hat{G} \mathbf{M}+\mathrm{j} \hat{B} \mathbf{M}
\end{aligned}
$$

where the $B_{n}$ susceptances are given by

$$
B_{n}= \begin{cases}\omega C_{n} ; \quad C_{n}=\varepsilon_{0} / \alpha_{n}, & \text { TM modes } \\ -\left(\omega L_{n}\right)^{-1} ; \quad L_{n}=\mu_{0} / \alpha_{n}, & \text { TE modes }\end{cases}
$$

with $\alpha_{n}$ being the attenuation constants of evanescent modes. Taking into account (10), the IE (1) can be rewritten as

$$
2 \mathbf{H}_{\mathrm{inc}}-\hat{G} \mathbf{M}=\mathrm{j} \hat{B} \mathbf{M} \text {. }
$$

In the following, it will be assumed that only the $n=0$ mode is propagative, with all the remaining ones being evanescent. This corresponds to the usual practical case in which the frequency range of interest lies within the monomode operation band of the waveguide. In this case, we have

$$
\begin{aligned}
& \mathbf{H}_{\mathrm{inc}}=Y_{0} E_{\mathrm{inc}} \mathbf{h}_{0} \\
& \hat{G} \mathbf{M}=2 Y_{0} T E_{\mathrm{inc}} \mathbf{h}_{0}
\end{aligned}
$$

where $E_{\text {inc }}$ is the electric-field amplitude of the incident wave and $T$ its transmission coefficient. The IE (12), thus, becomes

$$
-2 Y_{0} E_{\mathrm{inc}} R \mathbf{h}_{0}=\mathrm{j} \hat{B} \mathbf{M}
$$

where $R$ is the reflection coefficient for the electric field, with $T=1+R$. Now, it is key to realize that, at resonance frequencies, the impinging mode is completely transmitted through the discontinuity and, therefore, at resonance frequencies, the corresponding resonant magnetic current patterns must satisfy the following IE in the aperture region:

$$
\hat{B} \mathbf{M}=\mathbf{0} \text {. }
$$

Next, we can write the $\hat{B}$ operator as

$$
\hat{B}=\omega \hat{C}-\omega^{-1} \hat{L}^{-1}
$$

where $\hat{C}$ and $\hat{L}^{-1}$ operators, given by

$$
\begin{aligned}
\hat{C} & =\sum_{n, \mathrm{TM}} 2 C_{n} \hat{p}_{n} \\
\hat{L}^{-1} & =\sum_{n, \mathrm{TE}} \frac{2}{L_{n}} \hat{p}_{n}
\end{aligned}
$$

represent the contributions of TM and TE evanescent modes to the susceptance operator, respectively. Introducing now (18) and (19) into the resonance condition (16) finally leads to

$$
\hat{L}^{-1} \mathbf{M}=\omega^{2} \hat{C} \mathbf{M} .
$$

This equation is a generalized eigenproblem whose eigenvalues are the square of the resonance angular frequencies and its eigenfunctions are the corresponding resonant magnetic current (or aperture field) profiles. The $\hat{L}^{-1}$ and $\hat{C}$ operators are Hermitian and positive definite. As a simple proof of the latter, consider that any magnetic current $\mathbf{M}$ defined over the apertures will excite evanescent fields, with some amount of time-average reactive electric (magnetic) energy stored in the fields of the TM (TE) evanescent modes. Since these timeaveraged electric and magnetic stored energies can be written as

$$
\begin{aligned}
W_{e} & =\frac{1}{4}\langle\mathbf{M}, \hat{C} \mathbf{M}\rangle \\
W_{m} & =\frac{1}{4 \omega^{2}}\left\langle\mathbf{M}, \hat{L}^{-1} \mathbf{M}\right\rangle
\end{aligned}
$$

it follows that $\hat{C}$ and $\hat{L}^{-1}$ are positive definite.

\section{SOLUTION OF THE EIGENPROBLEM}

As it is customary when dealing with IEs, the unknown $\mathbf{M}$ value in (20) is now expanded into a set of basis functions

$$
\mathbf{M}=\sum_{i=1}^{N} a_{i} \mathbf{m}_{i}
$$

so that (20) is turned into the following standard Hermitian definite matrix eigenproblem:

$$
\underline{\underline{L}}^{-1} \cdot \underline{a}=\omega^{2} \underline{\underline{C}} \cdot \underline{a}
$$

with $\underline{a}$ being the column vector containing the coefficients of the magnetic current expansion and where $\underline{\underline{L}}^{-1}$ and $\underline{\underline{C}}$ matrices are

$$
\begin{aligned}
\underline{\underline{C}} & =\sum_{n, \mathrm{TM}} 2 C_{n} \underline{\underline{p_{n}}} \\
\underline{\underline{L}}^{-1} & =\sum_{n, \mathrm{TE}} \frac{2}{L_{n}} \underline{\underline{p_{n}}}
\end{aligned}
$$


with the entries of the $\underline{\underline{p_{n}}}$ matrices given by

$$
p_{n, i j}=\left\langle\mathbf{m}_{i}, \hat{p}_{n} \mathbf{m}_{j}\right\rangle=\left\langle\mathbf{m}_{i}, \mathbf{h}_{n}\right\rangle\left\langle\mathbf{h}_{n}, \mathbf{m}_{j}\right\rangle .
$$

The solution of the generalized eigenproblem (24) can be carried out by some of the many reliable linear algebra packages; for instance [43].

At this point, it should be noted that the $\hat{L}^{-1}$ and $\hat{C}$ operators, as well as the $\underline{\underline{L}}^{-1}$ and $\underline{\underline{C}}$ matrices, are not frequencyindependent. The reason lies in the frequency dependence of the attenuation constants (dispersion) of evanescent modes, which affects the $L_{n}$ and $C_{n}$ modal inductances and capacitances defined in (11). However, this dispersive behavior of evanescent modes is relatively smooth except in a narrow frequency band around their cutoff frequency, which leads to a relatively weak frequency dependence of the matrices in the eigenproblem unless working at frequencies close to the upper bound of the monomode band. The idea is then to compute the matrices in (24) at a convenient value of frequency (e.g., at the center of the frequency band of interest) and solve the eigenproblem to obtain the resonance frequencies that fall within the monomode band. The eigenvalues thus obtained will give resonance frequencies that are only approximate, although, in general, it is found that the corresponding resonance magnetic current profiles (eigenvectors) form a quite suitable set of basis functions to expand with good accuracy the actual magnetic current excited by the impinging wave over all (or most of) the monomode frequency band. In consequence, in an MoM solution of the original IE (1) for the discontinuity problem, these resonant profiles can be advantageously used as basis functions. The main benefit would come from the reduction of the size of the algebraic system to be solved to just the number of resonances found within the monomode band. Depending on the number of apertures in the discontinuity and their geometry, this reduction can be very significant.

Let $\mathbf{M}_{j}$ be the obtained resonant profiles, written as

$$
\mathbf{M}_{j}=\sum_{i=1}^{N} a_{i j} \mathbf{m}_{i}, \quad j=1, \ldots, N_{\mathrm{r}}
$$

where $a_{i j}$ are the entries of matrix $\underline{a}$ formed out of the eigenvectors of (24) by columns. The change of basis from the original set of functions (23) to the resonant profiles is carried out by applying the following usual transformation to the already computed $\underline{\underline{p_{n}}}$ matrices:

$$
\underline{\underline{\tilde{p}_{n}}}=\underline{\underline{a^{\dagger}}} \cdot \underline{\underline{p_{n}}} \cdot \underline{\underline{a}}
$$

where the dagger denotes the conjugate transpose.

In order to illustrate the performance of the resonant profiles as basis functions, a first numerical example is considered in Fig. 2, which shows the magnitude and phase of the reflection coefficient for a three-slit discontinuity in a rectangular waveguide (see Fig. 2(a) and its inset for the structure parameters). Solid lines represent the values obtained using an in-house MoM code with subsectional rooftop basis functions over a rectangular mesh of the slits. Numerical results obtained with software CST Microwave Studio have also been added

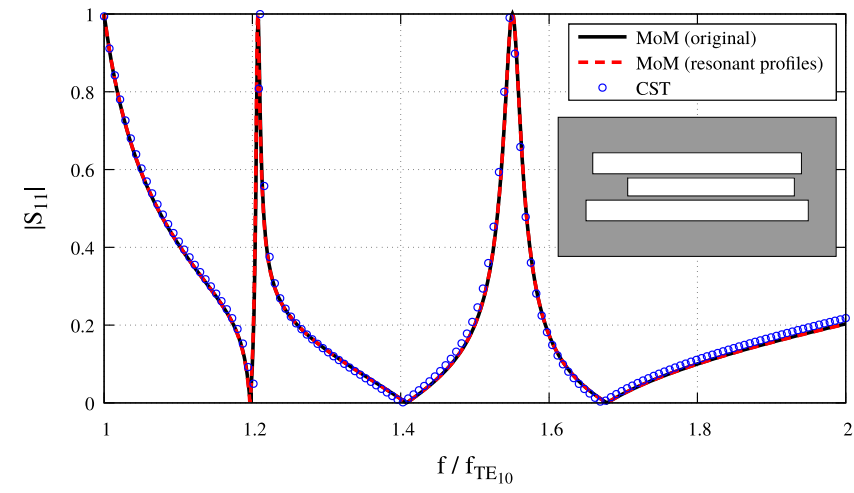

(a)

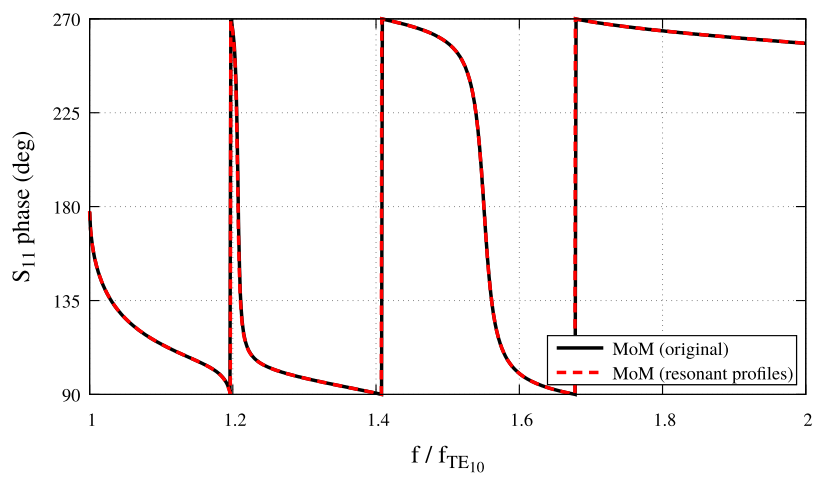

(b)

Fig. 2. (a) Magnitude and (b) phase of the reflection coefficient for a threeslit discontinuity in a rectangular waveguide. Waveguide dimensions: $a=2 b$. Slit lengths: $w_{x 1}=0.75 a, w_{x 2}=0.6 a$, and $w_{x 3}=0.8 a$. Slit widths: $w_{y 1}=$ $0.075 a, w_{y 2}=0.065 a$, and $w_{y 3}=0.075 a$. Vertical positions: $y_{1}=0.085 a$, $y_{2}=0$, and $y_{3}=-0.085 a$. Horizontal offset: $x_{1}=0, x_{2}=0.05 a$, and $x_{3}=0$.

for validation purposes. Three resonances (reflection nulls) can be observed within the monomode band at $f_{1}=1.20 f_{\mathrm{TE}_{10}}$, $f_{2}=1.41 f_{\mathrm{TE}_{10}}$, and $f_{3}=1.68 f_{\mathrm{TE}_{10}}$, with $f_{\mathrm{TE}_{10}}$ being the cutoff frequency of the $\mathrm{TE}_{10}$ mode. Alternatively, the same set of basis functions is used to evaluate the $\underline{\underline{L}}^{-1}$ and $\underline{\underline{C}}$ matrices at the center of the monomode band ( $f \equiv 1.5 f_{\mathrm{TE}_{10}} \overline{\bar{C}}$ ). The solution of the resulting eigenproblem (24) provides three eigenvalues within the considered band, located at $f_{1}=$ $1.15 f_{\mathrm{TE}_{10}}, f_{2}=1.40 f_{\mathrm{TE}_{10}}$, and $f_{3}=1.70 f_{\mathrm{TE}_{10}}$, together with their corresponding eigenvectors (magnetic current resonant profiles). A color map of the magnitude of the aperture electric field for the three resonant profiles is shown in Fig. 3. As mentioned earlier, the eigenvalues are approximate values of the resonance frequencies, but it is interesting to note that the second one is already very close to the MoM "exact" value, since it lies relatively close to the reference frequency at which the eigenproblem matrices were computed. If the three resonant profiles thus obtained are now taken as basis functions in the MoM, we obtain the dashed-line curves in Fig. 2. An excellent agreement with the original MoM is found all along the monomode band both in magnitude and phase.

It should be pointed out that the proposed formulation (and thus the circuit models derived from it in Section IV) only deals with zero-thickness discontinuities. However, different 


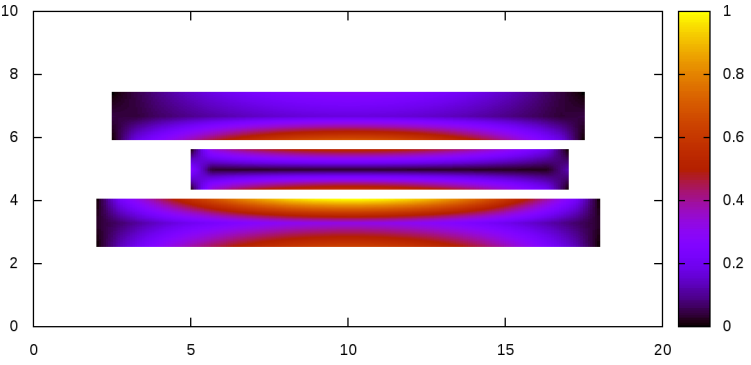

(a)

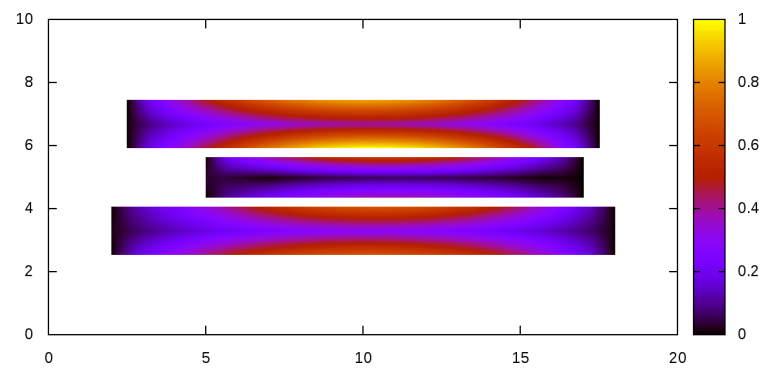

(b)

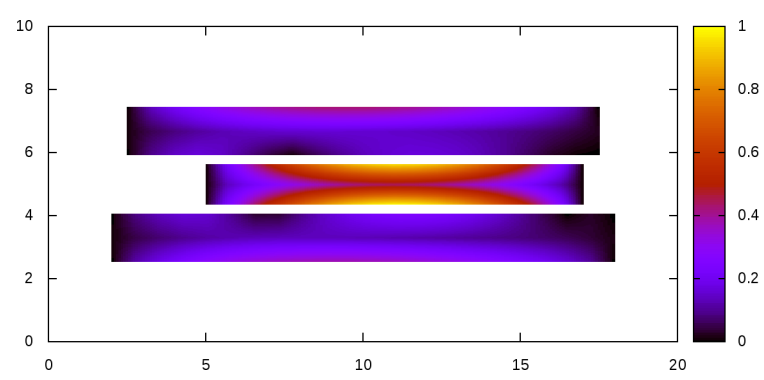

(c)

Fig. 3. Magnitude of the aperture electric field of the resonant profile obtained for the (a) first, (b) second, and (c) third resonance of the three-slits discontinuity previously analyzed in Fig. 2 after solving the eigenproblem at the middle frequency of the monomode band. These resonant profiles are used as basis functions in the MoM to obtain the dashed curves in Fig. 2.

comparisons with numerical results for finite-thickness irises obtained using commercial simulators (not included here) indicate that the effects of the finite thickness are well approximated by our results for practical values of the thickness. As an example, for the structure analyzed in Fig. 2, a resonance frequency shift of about $1 \%$ is found for a 0.5 -mm-thick screen in a waveguide with $a=20 \mathrm{~mm}$.

\section{ORThogonality And CiRCUit Model}

Let us assume that we have already solved the eigenproblem (24) using the matrices computed at a given frequency value, and we have thus obtained a set of $N_{\mathrm{r}}$ resonances within the frequency band of interest with associated magnetic current profiles $\left\{\mathbf{M}_{i}\right\}_{i=1, \ldots, N_{\mathrm{r}}}$. Using these resonant profiles as basis functions, the unknown magnetic current at the apertures is written as

$$
\mathbf{M}=\sum_{i=1}^{N_{\mathrm{r}}} b_{i} \mathbf{M}_{i} .
$$

The application of the MoM to the IE in (15), assuming that $E_{\text {inc }}=1$ without loss of generality, leads to the following system of algebraic equations:

$$
-2 Y_{0} R\left\langle\mathbf{M}_{j}, \mathbf{h}_{0}\right\rangle=\mathrm{j} \sum_{i=1}^{N_{\mathrm{r}}}\left\langle\mathbf{M}_{j}, \hat{B} \mathbf{M}_{i}\right\rangle b_{i} .
$$

As our eigenproblem is Hermitian definite, the eigenvectors corresponding to different eigenvalues are orthogonal with respect to both $\hat{L}^{-1}$ and $\hat{C}$, and therefore,

$$
\left\langle\mathbf{M}_{j}, \hat{L}^{-1} \mathbf{M}_{i}\right\rangle=\left\langle\mathbf{M}_{j}, \hat{C} \mathbf{M}_{i}\right\rangle=0 \text { for } i \neq j .
$$

In other words, both the $\underline{\underline{L}}^{-1}$ and $\underline{\underline{C}}$ matrices are diagonal in the basis of the magnetic-current resonant profiles. Rigorously speaking, this orthogonality relation only holds for the single frequency at which the eigenproblem was solved. However, due to the relatively smooth frequency dependence of the $\hat{B}$ operator, it seems reasonable to expect that these resonant profiles remain quasi-orthogonal within the monomode band of interest, and therefore, we will next assume that (32) is a good approximation in this frequency band. Using the modal expansion of the electric field at the discontinuity plane, it is also found that

$$
1+R=T=\left\langle\mathbf{h}_{0}, \mathbf{M}\right\rangle=\sum_{i=1}^{N_{\mathrm{r}}}\left\langle\mathbf{h}_{0}, \mathbf{M}_{i}\right\rangle b_{i}=\sum_{i=1}^{N_{\mathrm{r}}} V_{i}
$$

where $V_{i}=\left\langle\mathbf{h}_{0}, \mathbf{M}_{i}\right\rangle b_{i}$ can be interpreted as a partial voltage drop associated with the $i$ th resonant profile. Taking into account these considerations, from (31)-(33), the following equation is reached:

$$
Y_{0}(1-R)-Y_{0} T=\mathrm{j}\left[\omega C_{i}(\omega)-\frac{1}{\omega L_{i}(\omega)}\right] V_{i}
$$

with

$$
C_{i}(\omega)=\frac{\left\langle\mathbf{M}_{i}, \hat{C} \mathbf{M}_{i}\right\rangle}{\left|\left\langle\mathbf{h}_{0}, \mathbf{M}_{i}\right\rangle\right|^{2}} \quad L_{i}(\omega)=\frac{\left|\left\langle\mathbf{h}_{0}, \mathbf{M}_{i}\right\rangle\right|^{2}}{\left\langle\mathbf{M}_{i}, \hat{L}^{-1} \mathbf{M}_{i}\right\rangle} .
$$

Note that, if $\underline{\underline{\tilde{C}}}$ and $\underline{\underline{L}}^{-1}$ represent the matrices in the basis of the resonant profiles, then (35) can directly be written in terms of their diagonal elements as

$$
C_{i}(\omega)=\frac{\tilde{C}_{i i}(\omega)}{\left|\left\langle\mathbf{h}_{0}, \mathbf{M}_{i}\right\rangle\right|^{2}} \quad L_{i}(\omega)=\frac{\left|\left\langle\mathbf{h}_{0}, \mathbf{M}_{i}\right\rangle\right|^{2}}{\tilde{L}_{i i}^{-1}(\omega)} .
$$

From a transmission-line model standpoint, the left-hand side of (34) is clearly identified as the difference between the current at both sides of the discontinuity. In consequence, (33) and (34) are fully consistent with the equivalent network shown in Fig. 4, which consists of $N_{\mathrm{r}}$ seriesconnected resonant $L C$ tanks whose $L$ and $C$ values are given by (35) or equivalently by (36). Actually, this circuit model can be regarded as a canonical Foster synthesis [8] with frequency-dependent elements. It could be objected that Foster's canonical form includes, in general, an additional $L C$ series resonator. However, it should be considered that, first, the topology of the equivalent circuit has not been qualitatively or heuristically proposed but derived from the IE formulation and, second, that such additional $L$ and $C$ series elements could naturally appear in our model as the degeneration of a $L C$ shunt tank with infinite value of $L$ or null value 


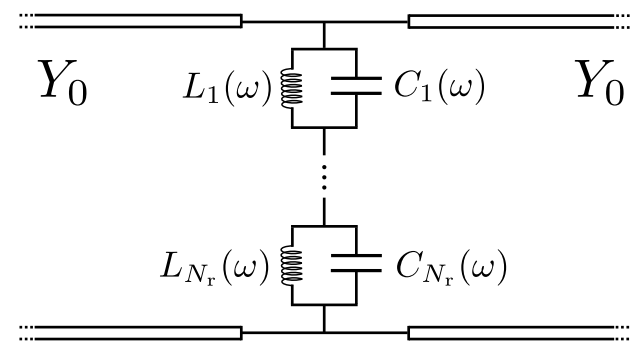

Fig. 4. Circuit model derived under the approximation that the eigenvectors keep their orthogonality in the frequency band of interest. The circuit has the topology of Foster's first canonical form but with frequency-dependent capacitances and inductances.

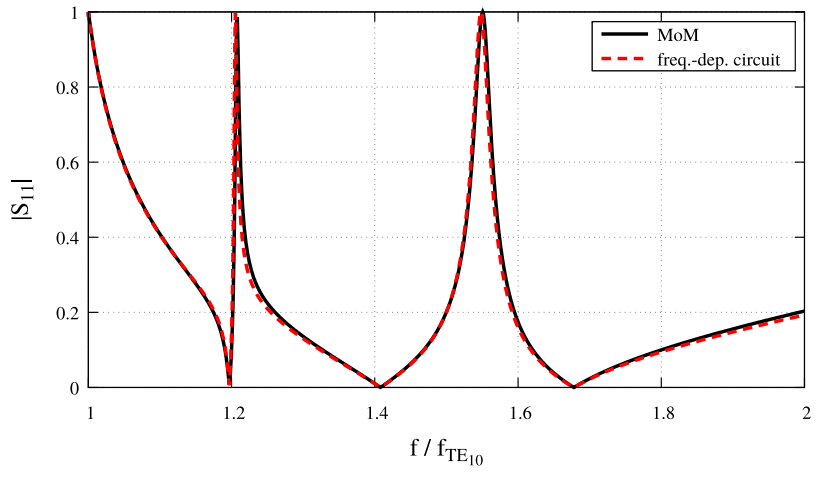

(a)

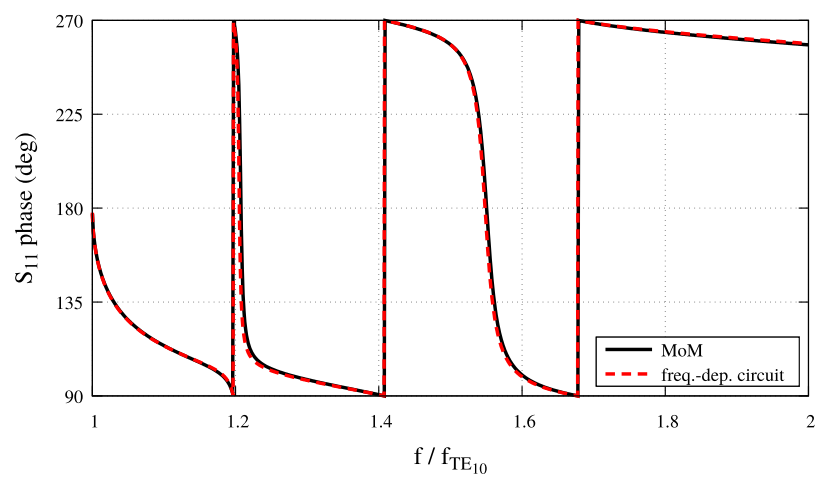

(b)

Fig. 5. (a) Magnitude and (b) phase of the reflection coefficient obtained using the MoM and using the Foster-like circuit model with frequencydependent elements shown in Fig. 4. Structure parameters: same as in Fig. 2.

of $C$ (namely, resonance frequencies at either zero or infinity). The derivation of this equivalent Foster's synthesis, both its topology and the values of the elements involved, is considered a relevant achievement of the present procedure.

The application of the previous circuit model approach to the structure considered in Fig. 2 is shown in Fig. 5, which shows the reflection coefficient provided by the circuit model together with the original MoM results. Although a close inspection of these plots reveals some slight deviations, it can be said that the performance of the circuit model is very good overall. In terms of computational effort, it is worth noting that, according to (35) or (36), the computation of each value of the circuit elements at each frequency value is similar to that of a single element of the Galerkin matrix.

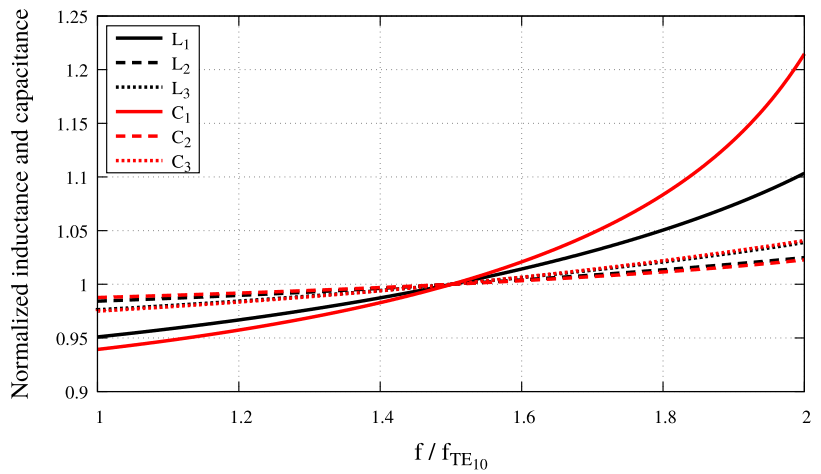

Fig. 6. Inductances and capacitances of the frequency-dependent circuit model for the structure previously analyzed in Fig. 5, computed using (35) for each of the three resonances. All the curves are normalized to their original values at the central frequency of the monomode band (see Table I).

TABLE I

Circuit Element VAlues

\begin{tabular}{|c|c|c||c|c|}
\cline { 2 - 5 } \multicolumn{1}{c|}{} & \multicolumn{2}{c||}{$L_{i}(\mathrm{nH})$} & \multicolumn{2}{c|}{$C_{i}(\mathrm{pF})$} \\
\cline { 2 - 5 } \multicolumn{1}{c|}{} & original & refined & original & refined \\
\hline$i=1$ & 0.3957 & 0.3824 & 0.8604 & 0.8233 \\
\hline$i=2$ & 4.694 & 4.678 & 0.04877 & 0.04864 \\
\hline$i=3$ & 3.536 & 3.577 & 0.04424 & 0.04478 \\
\hline
\end{tabular}

Although Fig. 5 shows a very good performance of the frequency-dependent circuit, a circuit model with frequencyindependent elements can be preferable for practical design purposes, even though some loss of accuracy should then be expected. In this regard, Fig. 6 shows the frequency dependence of the six circuit elements used in Fig. 5. All the capacitances and inductances are normalized to their original values obtained at the center frequency of the monomode band (the reference frequency at which the eigenvalue problem was solved). These values are shown in the columns labeled "original" in Table I for a waveguide with $a=20 \mathrm{~mm}$ $\left(f_{\mathrm{TE}_{10}} \approx 7.5 \mathrm{GHz}\right)$. It can be observed that the frequency dependence of the capacitances and inductances associated with the second and third resonances is very weak and a bit stronger for the first resonance. In any case, the observed frequency behavior of these circuit elements is quite smooth, and even for the worst case (the capacitance corresponding to the first resonance $C_{1}$ ), its variations within the monomode band are within $20 \%$ of its value at the center frequency. Therefore, as a first proposal for a frequency-independent circuit, we can just take the values of the circuit elements at the reference frequency at which the eigenproblem was solved (these values are already available after solving the eigenproblem, since the $\underline{\underline{L}}^{-1}$ and $\underline{C}$ matrices have already been computed at that frequency value). The results obtained using this frequencyindependent circuit model are shown in Fig. 7 (dashed curve labeled "original circuit"). The agreement with the MoM results is reasonable, although some deviations can be clearly observed, as expected. In particular, the deviations are more pronounced in the first resonance, which is clearly shifted toward lower frequencies. The second and third resonances are less affected, as expected since they are closer to the reference frequency $\left(1.5 f_{\mathrm{TE}_{10}}\right)$. 


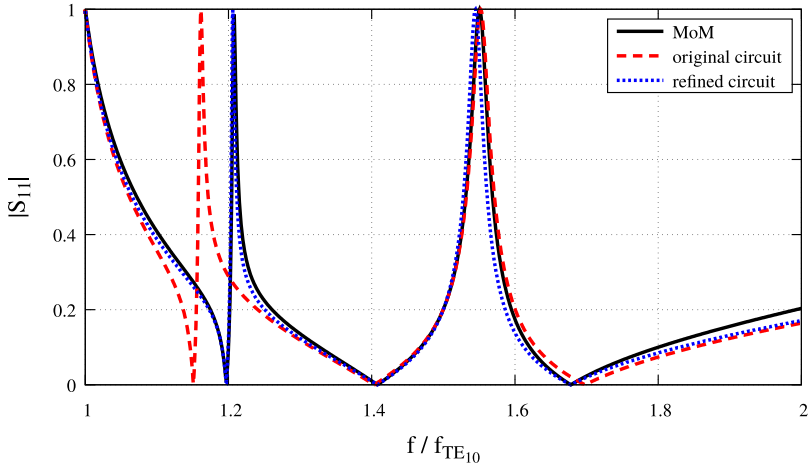

(a)

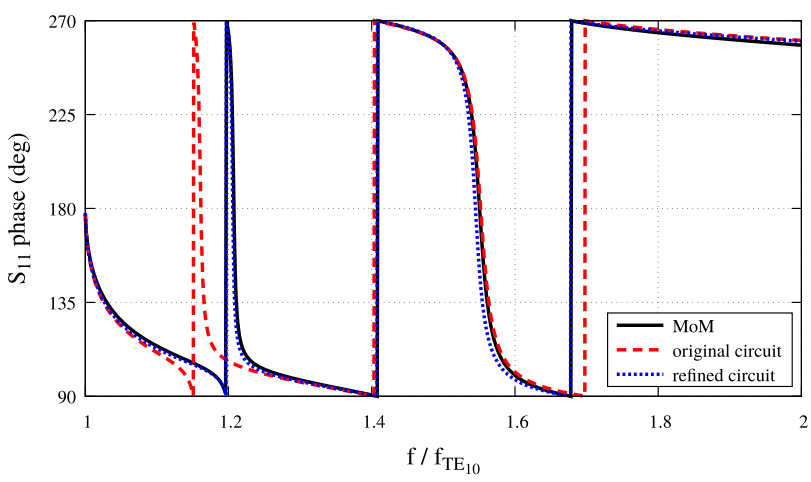

(b)

Fig. 7. (a) Magnitude and (b) phase of the reflection coefficient obtained using the MoM and using the obtained Foster canonical circuit models with frequency-independent elements, for the same discontinuity previously analyzed in Figs. 2-6. The curve labeled "original circuit" corresponds to the results obtained using the circuit elements computed at the original reference frequency $f / f_{\mathrm{TE}_{10}}=1.5$. The curve labeled "refined circuit" is obtained using the circuit elements that result after applying the iterative refinement (37)-(39). The numerical values of the elements of both circuits for $a=20 \mathrm{~mm}$ are shown in Table I.

Although the results provided by the above-mentioned circuit with frequency-independent elements may be quite reasonable, in many practical cases, they will not be accurate enough. However, the frequency-independent equivalent circuit can be taken as a starting point for an iterative strategy designed to refine the values of the circuit elements. Next, we propose a very simple procedure that, in our experience, has proved to be very efficient and reliable. Let $\omega_{i}^{(0)}$ denote the approximate angular frequency of the $i$ th resonance already obtained after solving the eigenproblem at the reference frequency. The first step is to compute the values of the associated inductance and capacitance at the approximate resonance frequency $\omega_{i}^{(0)}$ using (35). These first refined values are denoted as $L_{i}^{(1)}$ and $C_{i}^{(1)}$. From these values, we can compute a new value of the resonance frequency, and so on. This iterative strategy can be summarized as

$$
\begin{aligned}
C_{i}^{(k)} & =C_{i}\left(\omega_{i}^{(k-1)}\right)=\frac{\left\langle\mathbf{M}_{i}, \hat{C}\left(\omega_{i}^{(k-1)}\right) \mathbf{M}_{i}\right\rangle}{\left|\left\langle\mathbf{h}_{0}, \mathbf{M}_{i}\right\rangle\right|^{2}} \\
L_{i}^{(k)}=L_{i}\left(\omega_{i}^{(k-1)}\right) & =\frac{\left|\left\langle\mathbf{h}_{0}, \mathbf{M}_{i}\right\rangle\right|^{2}}{\left\langle\mathbf{M}_{i}, \hat{L}^{-1}\left(\omega_{i}^{(k-1)}\right) \mathbf{M}_{i}\right\rangle}
\end{aligned}
$$

$$
\omega_{i}^{(k)}=\frac{1}{\sqrt{L_{i}^{(k)} C_{i}^{(k)}}}
$$

where it should be noted that the resonant magnetic-current profile $\mathbf{M}_{i}$ used throughout the iterative process is always the same one originally obtained at the reference frequency $\omega_{i}^{(0)}$. In our experience, in most cases, this simple strategy converges conveniently within reasonable tolerance margins after just a few iterations (in our computations, we typically require a relative error below $0.1 \%$ between two consecutive iterations). By applying this iterative strategy to the previously considered three-slit discontinuity, we obtain the refined values of the circuit elements shown in Table I. The correction introduced with respect to the original values is more noticeable in the case of the first resonance, as expected since the shift with respect to the resonance frequencies predicted by the MoM results is less pronounced for the second and third resonances. The reflection spectrum provided by this refined circuit model is also shown in Fig. 7, labeled as "refined circuit." As it can be observed, the iterative strategy provides a circuit model that mostly corrects the deviations of the previous circuit and, although not quite as accurate as the circuit with frequencydependent elements, the comparison with MoM results is very satisfactory.

As an additional relevant comment it should be pointed out that, with independence of the circuit model interpretation, the above-proposed iterative scheme can be interesting by itself as an efficient and systematic strategy to compute the resonance frequencies of a given multiresonant iris waveguide discontinuity.

\section{EXCitation OF THE RESONANCES}

The degree of excitation of the resonances is an interesting and relevant issue that finds a very appropriate framework of discussion within the proposed formulation thanks to the possible interpretation in terms of the obtained circuit models. To illustrate the discussion, we will consider a symmetric variation of the three-slit discontinuity analyzed in Section IV (see Fig. 8 for the structure parameters). After solving the associated eigenvalue problem, three resonances are obtained within the monomode band at $f_{1}=1.127 f_{\mathrm{TE}_{10}}, f_{2}=$ $1.332 f_{\mathrm{TE}_{10}}$, and $f_{3}=1.686 f_{\mathrm{TE}_{10}}$. However, the MoM results in Fig. 8(a) show only two resonances. The reason of this apparent disagreement lies in the parity of the vertical component of the aperture field profile of the first resonance, which is odd with respect to the horizontal middle plane of the discontinuity and, therefore, it is not excited by the impinging $\mathrm{TE}_{10}$ mode. When using the obtained resonant profiles as basis functions in the MoM, the excitation coefficient of the first resonant profile is negligible, because its projection over the impinging wave, given by $\left\langle\mathbf{h}_{0}, \mathbf{M}_{1}\right\rangle=\left\langle\mathbf{e}_{0}, \mathbf{E}_{\mathrm{a}, 1}\right\rangle$ in the formulation, is virtually zero.

It is then very convenient to have a figure of merit to measure the degree of excitation of the different resonant profiles so that those resonances that are very weakly excited (or not excited at all) can be discarded. A first idea could be to use the magnitude of the projection $\left|\left\langle\mathbf{h}_{0}, \mathbf{M}\right\rangle\right|$ itself, but this choice has the disadvantage that its value obviously 


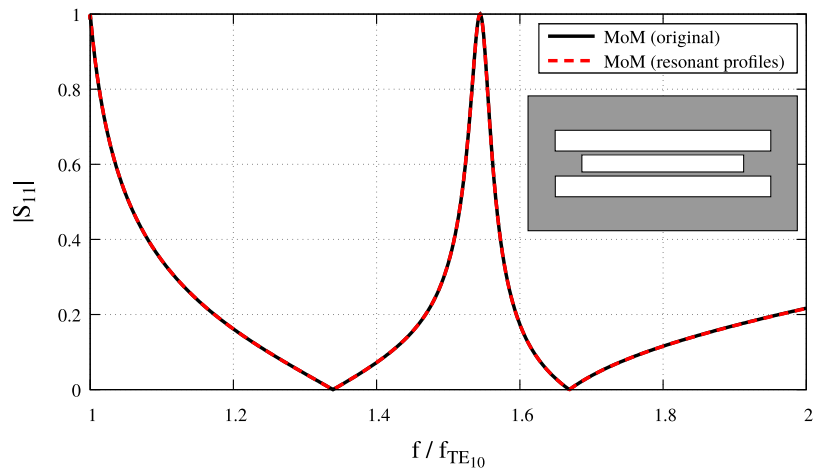

(a)

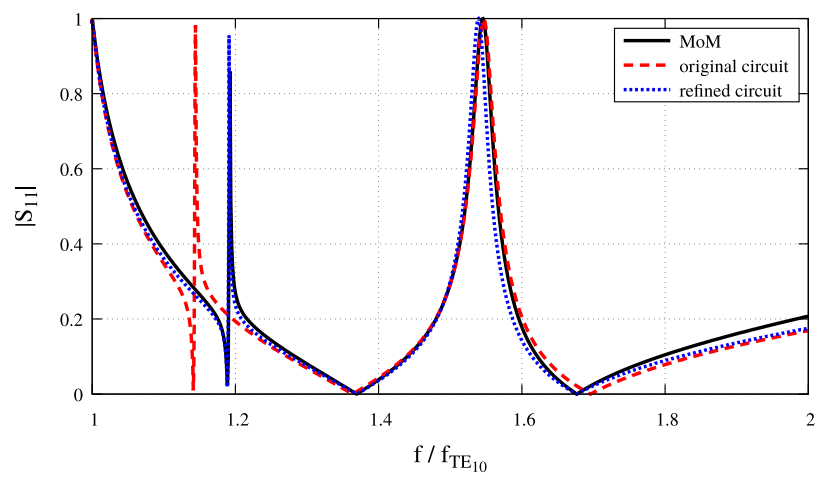

(b)

Fig. 8. Magnitude of the reflection coefficient. (a) Symmetric three-slit discontinuity. Waveguide dimensions: $a=2 b$. Slit lengths: $w_{x 1}=0.8 a$, $w_{x 2}=0.6 a$, and $w_{x 3}=w_{x 1}$. Slit widths: $w_{y 1}=0.075 a, w_{y 2}=0.065 a$ and $w_{y 3}=w_{y 1}$. Vertical positions: $y_{1}=0.085 a, y_{2}=0$, and $y_{3}=-y_{1}$. Horizontal offset: $x_{1}=x_{2}=x_{3}=0$. (b) Nonsymmetric structure derived from the previous structure but now with $w_{x 1}=0.775 a$ and $w_{x 3}=0.8 a$.

depends on the specific normalization of the resonant profiles. Other figures should then be proposed for that purpose. In this way, it is found that the canonical circuit-model approach naturally provides a convenient and intuitive choice. For a given resonance, we can define an "intrinsic impedance" of its associated resonator in the circuit as

$$
Z_{i}=\sqrt{\frac{L_{i}}{C_{i}}}=\frac{\left|\left\langle\mathbf{h}_{0}, \mathbf{M}_{i}\right\rangle\right|^{2}}{\sqrt{\left\langle\mathbf{M}_{i}, \hat{C} \mathbf{M}_{i}\right\rangle\left\langle\mathbf{M}_{i}, \hat{L}^{-1} \mathbf{M}_{i}\right\rangle}} .
$$

Following a circuit-theory rationale, the ratio of this intrinsic impedance to the characteristic impedance of the impinging mode would provide a good measure of the degree of excitation of the resonance. If this ratio is very small, the associated resonator in the circuit model would practically behave as a short circuit and will not have any significant effect in the spectrum, except at frequencies very close to its resonance frequency. In our experience, resonances with a ratio under $10^{-5}$ can be safely discarded. For the symmetric discontinuity analyzed in Fig. 8(a), the ratio of impedances for the first resonance is virtually zero (our numerical calculation yields a value less than $10^{-20}$ ).

If the symmetry of the structure considered in Fig. 8(a) is now broken by slightly changing the length of one of the off-centered slits, the reflection spectrum shown in Fig. 8(b) is obtained. This new spectrum is very similar to the one in Fig. 8(a) except for the appearance of the first resonance that was missing in the symmetric structure. The values of the intrinsic impedances of the three resonators obtained after solving the eigenvalue problem at the middle frequency of the monomode band are $Z_{1}=0.008 Z_{\mathrm{TE}_{10}}, Z_{2}=0.65 Z_{\mathrm{TE}_{10}}$, and $Z_{3}=0.57 Z_{\mathrm{TE}_{10}}$. Since the impedance of the first resonator is very small in comparison with the characteristic impedance $Z_{\mathrm{TE}_{10}}$, its associated resonance is very narrow (which is clearly related to the fact that the corresponding resonant field profile is quasi-odd with respect to the horizontal middle plane). It can also be observed that the reflection zero is very closely followed by a total reflection peak. This Fano-like behavior can, as well, be easily explained in terms of the circuit model. First of all, the existence of the reflection peak can be seen as a direct consequence of Foster's reactance theorem, which, in this context, guarantees that there is always a reflection peak between two reflection zeroes. The reason that this reflection peak appears so close above the resonance (reflection zero) frequency can be found in the small value of the resonator intrinsic impedance. Just above the first resonance frequency, the first resonator presents a high capacitive reactance that very quickly falls down to almost negligible values as frequency is slightly increased, thus canceling the inductive reactance of the other two resonators at some higher frequencies but very close to the reflection zero. This series resonance clearly results in an short-circuit behavior of the discontinuity that causes total reflection of the impinging wave. Similar discussions could also be applied to other structures, which can be very beneficial to understand the physical basis of some complex transmission/reflection spectra and thus help to guide design and/or optimization procedures.

\section{Further Results And ReFinement}

In this section, the above-mentioned formulation is applied to a more complex discontinuity that comprises five different nonsymmetrical slots [see the inset in Fig. 9(a)]. Also, a further refinement is proposed that introduces a correction in the resonant profiles. Thus, Fig. 9 shows a comparison between the reflection coefficient computed by the direct application of the MoM and by using the equivalent circuits, both with frequency-dependent and frequency-independent elements (only the magnitude of the reflection coefficient is shown, since a very similar agreement is also found in the phase results for all the comparisons). The results provided by the frequency-dependent circuit in Fig. 9(a) are overall very good, but the deviations with respect to MoM results, although small, are more noticeable than those found for the threeslit case in Fig. 5(a), especially toward the high-frequency portion of the monomode band. Clearly, the reason for this deviation can be found in the frequency dependence of the eigenvalue problem, which causes the resonant profiles not to be strictly orthogonal at frequencies sufficiently higher than the reference frequency at which they were obtained. Concerning the performance of the frequency-independent circuit models, it is observed in Fig. 9(b) that the original circuit obtained from the solution of the eigenproblem at $f=1.5 f_{\mathrm{TE}_{10}}$ 
TABLE II

Circuit Element Values and Resonance Frequencies (Five Slits)

\begin{tabular}{|c|c|c|c||c|c|c||c|c|c|c|}
\cline { 2 - 11 } \multicolumn{1}{c|}{} & \multicolumn{3}{c||}{$L_{i}(\mathrm{nH})$} & \multicolumn{3}{c||}{$C_{i}(\mathrm{pF})$} & \multicolumn{3}{c|}{ normalized resonance frequencies } \\
\cline { 2 - 11 } \multicolumn{1}{c|}{} & original & refined & new prof. & original & refined & new prof. & original & refined & new prof. & MoM \\
\hline$i=1$ & 0.6089 & 0.5915 & 0.6652 & 0.4927 & 0.4717 & 0.4202 & 1.226 & 1.271 & 1.270 & 1.270 \\
\hline$i=2$ & 0.04481 & 0.04471 & 0.05681 & 5.290 & 5.274 & 4.150 & 1.379 & 1.383 & 1.383 & 1.383 \\
\hline$i=3$ & 0.2760 & 0.2762 & 0.2761 & 0.7089 & 0.7092 & 0.7095 & 1.518 & 1.517 & 1.517 & 1.517 \\
\hline$i=4$ & 0.7620 & 0.7675 & 0.9616 & 0.2056 & 0.2070 & 0.1652 & 1.697 & 1.685 & 1.685 & 1.685 \\
\hline$i=5$ & 4.122 & 4.268 & 3.999 & 0.02863 & 0.02998 & 0.03173 & 1.955 & 1.877 & 1.885 & 1.883 \\
\hline
\end{tabular}

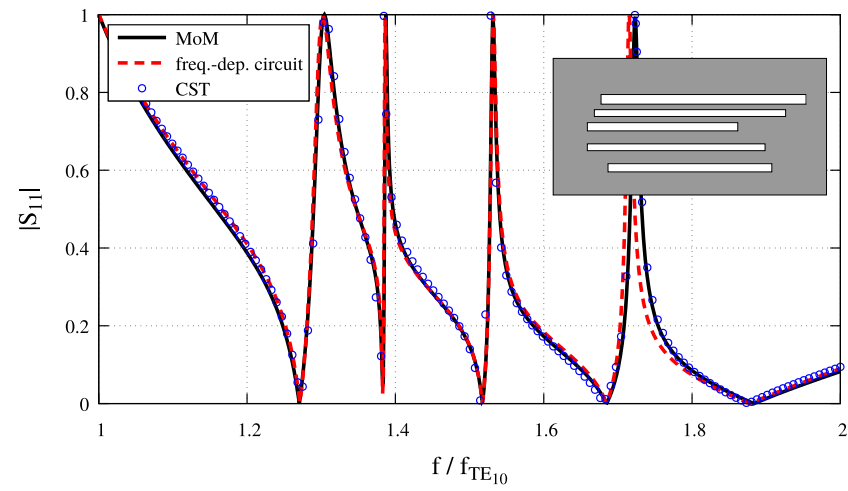

(a)

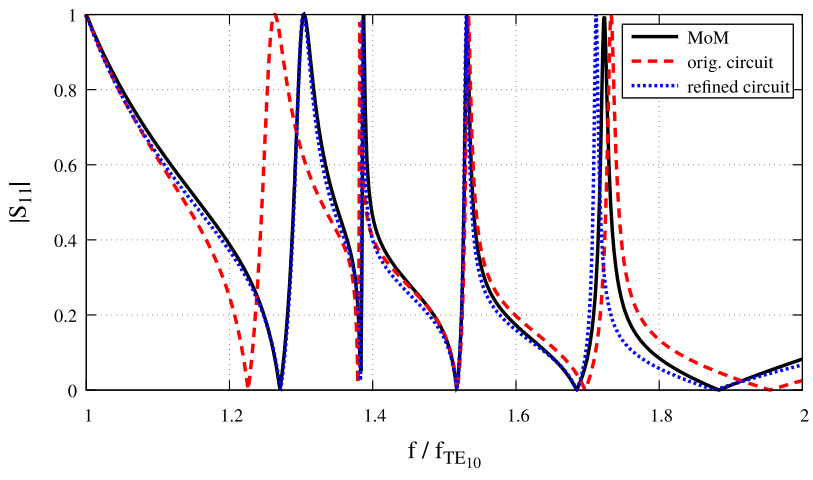

(b)

Fig. 9. Comparison between the reflection coefficients obtained using the MoM and using the Foster-like circuit models with (a) frequency-dependent and (b) frequency-independent elements. The inset in (a) shows a sketch of the considered five-slits discontinuity, with the following parameters. Waveguide dimensions: $a=2 b$; slit lengths: $w_{x 1}=0.75 a, w_{x 2}=0.7 a, w_{x 3}=0.55 a$, $w_{x 4}=0.65 a$, and $w_{x 5}=0.6 a$; slit widths: $w_{y 1}=0.035 a, w_{y 2}=0.025 a$, $w_{y 3}=0.03 a, w_{y 4}=0.025 a$, and $w_{y 5}=0.03 a$; vertical positions: $y_{1}=0.1 a$, $y_{2}=0.05 a, y_{3}=0, y_{4}=-0.075 a$, and $y_{5}=-0.15 a$; and horizontal offsets: $x_{1}=0.05 a, x_{2}=0, x_{3}=-0.1 a, x_{4}=-0.05 a$, and $x_{5}=0$.

still yields somewhat reasonable results, although it clearly shows the expected deviations as the frequency moves away from the central reference value. The refined circuit obtained after applying the iterative refinement scheme proposed in Section V satisfactorily corrects these deviations for the lower and intermediate frequencies, but, like the frequencydependent circuit, it still exhibits some more noticeable differences with respect to MoM at higher frequencies. The values of the capacitances and inductances of both the original and the refined circuit model are shown in Table II (the largest dimension of the waveguide is once again set to $a=20 \mathrm{~mm}$ ),

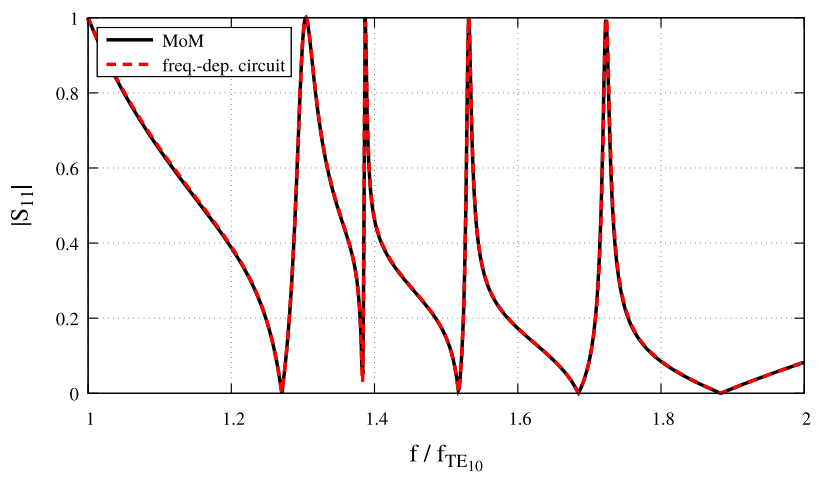

(a)

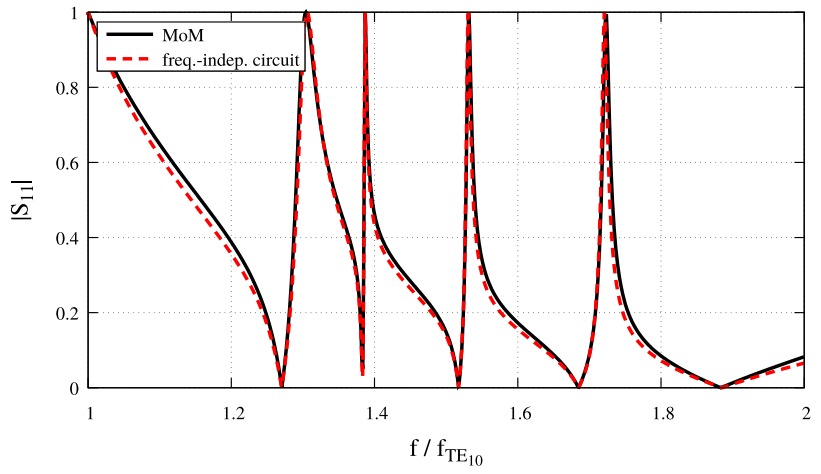

(b)

Fig. 10. Comparison between the original MoM data and the results obtained using the new resonant profiles provided by (41), for the same five-slits discontinuity previously analyzed in Fig. 9. (a) Circuit model with frequencydependent elements. (b) Circuit model with frequency-independent elements.

together with the resonance frequencies predicted by the circuit models and obtained with the MoM (the values of the resonance frequencies are normalized to $f_{\mathrm{TE}_{10}}$ ).

In order to overcome these limitations to some extent, we can introduce a correction in the resonant profiles as follows. At each value of the resonance frequency $\omega_{i}$ obtained after the iterative refinement, the eigenproblem is solved using the original resonant profiles as basis functions. Namely, if $N_{\mathrm{r}}$ is the number of resonances, we solve the following $N_{\mathrm{r}}$ matrix eigenproblems of size $N_{\mathrm{r}} \times N_{\mathrm{r}}$ :

$$
\underline{\underline{\tilde{L}}}^{-1}\left(\omega_{i}\right) \cdot \underline{b}=\omega^{2} \underline{\underline{\tilde{C}}}\left(\omega_{i}\right) \cdot \underline{b}, \quad \text { for } i=1, \ldots, N_{\mathrm{r}} .
$$

Thus, the $i$ th eigenvector of the $i$ th eigenproblem is taken as the new resonant profiles corresponding to the $i$ th resonance. 
When these new resonant profiles are used in (35) to compute the frequency-dependent elements, we obtain the reflection coefficient results as shown in Fig. 10(a). As it can be observed, the agreement with the original MoM is sensibly improved with respect to Fig. 9(a), and now, both curves are practically indistinguishable. Alternatively, in order to have a circuit with frequency-independent elements, we can use the new resonant profiles to evaluate the $i$ th capacitance and inductance using (35) at the corresponding $\omega_{i}$ resonance frequency (the refined values of the resonance frequency previously obtained from the iterative strategy). The values of the circuit elements thus obtained for the five-slit discontinuity under consideration, as well as the corresponding resonance frequencies, are also included in Table II ("new prof." columns). In Fig. 10(b), the values of the reflection coefficient magnitude provided by this frequency-independent circuit are compared with the original MoM results. The agreement between both sets of data is quite satisfactory, and once again a noticeable improvement is observed with respect to Fig. 9(b).

\section{CONCLUSION}

A novel formulation for the determination of the response of multiresonant irislike discontinuities in a hollow-pipe waveguide has been presented. The resonance frequencies are obtained from the eigenvalues of a properly posed eigenvalue problem. The associated eigenvectors are the resonant electricfield profiles on the aperture domains and can be used as a convenient basis in a moment-method formulation. The orthogonality properties of the eigenvectors can be exploited to derive an equivalent circuit in the form of a canonical Foster topology although involving frequency-dependent capacitors and inductors. Furthermore, using iterative refinement techniques, the values of the circuit components of a frequencyindependent Foster topology can be systematically obtained from the proposed formulation, rather than from the full electrical response of the multiresonant structure, as it has been done in other works. The agreement between the full-wave results and the results generated using the frequency-dependent and the optimized frequency-independent equivalent Foster networks is very good over a very wide frequency band.

\section{REFERENCES}

[1] R. G. Fellers and R. T. Weidner, "Broad-band wave-guide admittance matching by use of irises," Proc. IRE, vol. 35, no. 10 pp. 1080-1085, Oct. 1947. [Online]. Available: http://ieeexplore.ieee. org/document/1697203/

[2] J. Hessel, G. Goubau, and L. R. Battersby, "Microwave filter theory and design," Proc. IRE, vol. 37, no. 9, pp. 990-1000, Sep. 1949. [Online]. Available: http://ieeexplore.iee.org/document/1698130/

[3] I. Reingold, J. L. Carter, and K. Garoff, "Single- and multi-iris resonant structures," Proc. IRE, vol. 40, no. 7, pp. 861-865, Jul. 1952. [Online]. Available: $\mathrm{http} / / /$ ieeexplore.ieee.org/document/4051060/

[4] A. J. Simmons, "Phase shift by periodic loading of waveguide and its application to broad-band circular polarization," IRE Trans. Microw. Theory Techn., vol. 3, no. 6, pp. 18-21, Dec. 1955. [Online]. Available: http://ieeexplore.ieee.org/document/1124986/

[5] D. Rosenberg and D. J. R. Stock, "Some results for thin-iris loaded periodic waveguides," IEEE Trans. Microw. Theory Techn., vol. 14, no. 3, pp. 145-153, Mar. 1966. [Online]. Available: http://ieeexplore. ieee.org/document/1126192/
[6] J. D. Rhodes, "Waveguide bandstop elliptic function filters," IEEE Trans. Microw. Theory Techn., vol. 20, no. 11, pp. 715-718, Nov. 1972. [Online]. Available: http://ieeexplore.ieee.org/document/1127859/

[7] T. E. Rozzi, "Equivalent network for interacting thick inductive irises," IEEE Trans. Microw. Theory Techn., vol. 20, no. 5, pp. 323-330, May 1972. [Online]. Available: http://ieeexplore.ieee. org/document/1127752/

[8] C. G. Montgomery, R. H. Dicke, and E. M. Purcell, Eds., Principles of Microwave Circuits. New York, NY, USA: McGraw-Hill, 1948. [Online]. Available: http://www.worldcat.org/title/principles-ofmicrowave-circuits-edited-by-cg-montgomery-rh-dicke-em-purcell-etc/ oclc/769453903

[9] N. Marcuvitz, Waveguide Handbook. New York, NY, USA: McGraw-Hill, 1951.

[10] J. S. Schwinger and D. S. Saxon, Discontinuities in Waveguides: Notes on Lectures by Julian Schwinger. New York, NY, USA Gordon and Breach, 1968. [Online]. Available: https://searchworks. stanford.edu/view/606345

[11] A. Wexler, "Solution of waveguide discontinuities by modal analysis," IEEE Trans. Microw. Theory Techn., vol. 15, no. 9, pp. 508-517, Sep. 1967. [Online]. Available: http://ieeexplore.ieee. org/document/1126521/

[12] S. W. Lee, W. R. Jones, and J. J. Campbell, "Convergence of numerical solutions of iris-type discontinuity problems," IEEE Trans. Microw. Theory Techn., vol. 19, no. 6, pp. 528-536, Jun. 1971. [Online]. Available: http://ieeexplore.ieee.org/document/1127569/

[13] M. S. Navarro, T. E. Rozzi, and Y. T. Lo, "Propagation in a rectangular waveguide periodically loaded with resonant irises," IEEE Trans. Microw. Theory Techn., vol. 28, no. 8, pp. 857-865, Aug. 1980. [Online]. Available: http://ieeexplore.ieee.org/document/1130183/

[14] H. Auda and R. F. Harrington, "A moment solution for waveguide junction problems," IEEE Trans. Microw. Theory Techn., vol. 31, no. 7, pp. 515-520, Jul. 1983. [Online]. Available: http://ieeexplore. ieee.org/document/1131539/

[15] R. W. Scharstein and A. T. Adams, "Galerkin solution for the thin circular iris in a TE 11 -mode circular waveguide," IEEE Trans. Microw. Theory Techn., vol. 36, no. 1, pp. 106-113, Jan. 1988. [Online]. Available: http://ieeexplore.ieee.org/document/3489/

[16] J. Bornemann and R. Vahldieck, "Characterization of a class of waveguide discontinuities using a modified $\mathrm{TE}_{m n}{ }^{x}$ mode approach," IEEE Trans. Microw. Theory Techn., vol. 38, no. 12, pp. 1816-1822, Dec. 1990. [Online]. Available: http://ieeexplore.ieee. org/document/64561/

[17] R. Yang and A. S. Omar, "Investigation of multiple rectangular aperture irises in rectangular waveguide using $\mathrm{TE}_{m n}{ }^{x}$-modes," IEEE Trans. Microw. Theory Techn., vol. 41, no. 8, pp. 1369-1374, Aug. 1993. [Online]. Available: http://ieeexplore.iee.org/document/241678/

[18] U. Papziner and F. Arndt, "Field theoretical computer-aided design of rectangular and circular iris coupled rectangular or circular waveguide cavity filters," IEEE Trans. Microw. Theory Techn., vol. 41, no. 3, pp. 462-471, Mar. 1993. [Online]. Available: http://ieeexplore.ieee.org/document/223746/

[19] T. Rozzi, A. Morini, F. Ragusini, and M. Mongiardo, "Analytical solution and frequency extraction of iris problems in waveguide by separation of variables," IEEE Trans. Microw. Theory Techn., vol. 45, no. 2, pp. 253-259, Feb. 1997. [Online]. Available: http://ieeexplore.ieee. org/document/557607/

[20] A. A. Kirilenko and L. P. Mospan, "Reflection resonances and natural oscillations of two-aperture iris in rectangular waveguide," IEEE Trans. Microw. Theory Techn., vol. 48, no. 8, pp. 1419-1421, Aug. 2000. [Online]. Available: http://ieeexplore.ieee.org/document/859492/

[21] I. Stevanovic, P. Crespo-Valero, and J. R. Mosig, "An integralequation technique for solving thick irises in rectangular waveguides," IEEE Trans. Microw. Theory Techn., vol. 54, no. 1, pp. 189-197, Jan. 2006.

[22] S. Marini, Á. Coves, V. E. Boria, and B. Gimeno, "Full-wave modal analysis of slow-wave periodic structures loaded with elliptical waveguides," IEEE Trans. Electron Devices, vol. 57, no. 2 , pp. 516-524, Feb. 2010. [Online]. Available: http://ieeexplore. ieee.org/document $/ 5353710 /$

[23] C. Carceller, P. Soto, V. Boria, and M. Guglielmi, "Capacitive obstacle realizing multiple transmission zeros for in-line rectangular waveguide filters," IEEE Microw. Wireless Compon. Lett., vol. 26, no. 10, pp. 795-797, Oct. 2016. [Online]. Available: http://ieeexplore.ieee. org/document/7572044/ 
[24] C. A. Leal-Sevillano, J. R. Montejo-Garai, J. A. Ruiz-Cruz, and J. M. Rebollar, "Wideband equivalent circuit for multi-aperture multiresonant waveguide irises," IEEE Trans. Microw. Theory Techn., vol. 64, no. 3, pp. 724-732, Mar. 2016. [Online]. Available: http://ieeexplore.ieee.org/document/7401135/

[25] R. Rodríguez-Berral, F. Mesa, and F. Medina, "Analytical multimodal network approach for 2-D arrays of planar patches/apertures embedded in a layered medium," IEEE Trans. Antennas Propag., vol. 63, no. 5, pp. 1969-1984, May 2015. [Online]. Available: http://ieeexplore.ieee.org/document/7047764/

[26] F. Mesa, R. Rodríguez-Berral, M. García-Vigueras, F. Medina, and J. R. Mosig, "Simplified modal expansion to analyze frequencyselective surfaces: An equivalent circuit approach," IEEE Trans. Antennas Propag., vol. 46, no. 3, pp. 1106-1111, Mar. 2016. [Online]. Available: http://ieeexplore.ieee.org/document/7368870/

[27] F. Mesa, R. Rodriguez-Berral, and F. Medina, "Equivalent circuit model: An efficient and physically-insightful tool for microwave engineering," IEEE Microw. Mag., to be published.

[28] G. Conciauro, M. Bressan, and C. Zuffada, "Waveguide modes via an integral equation leading to a linear matrix eigenvalue problem," IEEE Trans. Microw. Theory Techn., vol. MTT-32, no. 11, pp. 1495-1504, Nov. 1984. [Online]. Available: http://ieeexplore.ieee. org/document/1132880/

[29] S. Cogollos et al., "Efficient modal analysis of arbitrarily shaped waveguides composed of linear, circular, and elliptical arcs using the BI-RME method," IEEE Trans. Microw. Theory Techn., vol. 51, no. 12, pp. 2378-2390, Dec. 2003. [Online]. Available: http://ieeexplore. ieee.org/document/1256769/

[30] R. F. Harrington and J. R. Mautz, "Theory of characteristic modes for conducting bodies," IEEE Trans. Antennas Propag., vol. AP-19, no. 5, pp. 622-628, Sep. 1971. [Online]. Available: http://ieeexplore. ieee.org/document/1139999/

[31] R. F. Harrington and J. R. Mautz, "Computation of characteristic modes for conducting bodies," IEEE Trans. Antennas Propag., vol. AP-19, no. 5, pp. 629-639, Sep. 1971. [Online]. Available: http://ieeexplore. ieee.org/document/1139990/

[32] M. Cabedo-Fabres, E. Antonino-Daviu, A. Valero-Nogueira, and M. F. Batalle, "The theory of characteristic modes revisited: A contribution to the design of antennas for modern applications," IEEE Antennas Propag. Mag., vol. 49, no. 5, pp. 52-68, Oct. 2007. [Online]. Available: http://ieeexplore.ieee.org/document/4395295/

[33] J. Ethier and D. A. McNamara, "Through the looking glass: A characteristic mode view of electromagnetic modeling \& design," in Proc. 14th Int. Symp. Antenna Technol. Appl. Electromagn. Amer. Electromagn. Conf. (ANTEM/AMEREM), Ottawa, ON, Canada, Jul. 2010, pp. 1-4. [Online]. Available: http://ieeexplore.iee.org/document/5552577/

[34] Y. Chen and C.-F. Wang, Characteristic Modes: Theory and Applications in Antenna Engineering. Hoboken, NJ, USA: Wiley, 2015.

[35] R. F. Harrington and J. R. Mautz, "Characteristic modes for aperture problems," IEEE Trans. Microw. Theory Techn., vol. 33, no. 6, pp. 500-505, Jun. 1985. [Online]. Available: http://ieeexplore. ieee.org/document/1133105/

[36] Y. Leviatan, "Low-frequency characteristic modes for aperture coupling problems," IEEE Trans. Microw. Theory Techn., vol. MTT-34, no. 11, pp. 1208-1213, Nov. 1986. [Online]. Available: http://ieeexplore.ieee. org/document/1133518/

[37] A. El-Hajj, K. Y. Kabalan, and R. F. Harrington, "Characteristic mode analysis of electromagnetic coupling through multiple slots in a conducting plane," IEE Proc. H-Microw., Antennas Propag., vol. 140, no. 6, pp. 421-425, Dec. 1993. [Online]. Available: http://link.aip.org/link/IPHPE7/v140/i6/p421/s1\&Agg=doi

[38] A. El-Hajj and K. Y. Kabalan, "Characteristic modes of a rectangular aperture in a perfectly conducting plane," IEEE Trans. Antennas Propag., vol. 42, no. 10, pp. 1447-1450, Oct. 1994. [Online]. Available: http://ieeexplore.ieee.org/document/320753/
[39] A. El-Hajj and K. Y. Kabalan, "Scattering from and penetration into a dielectric-filled conducting cylinder with multiple apertures," IEEE Trans. Electromagn. Compat., vol. 36, no. 3, pp. 196-200, Aug. 1994. [Online]. Available: http://ieeexplore.ieee.org/document/305454/

[40] K. Y. Kabalan, A. El-Hajj, S. Khoury, and A. Rayes, "Electromagnetic coupling to conducting objects behind apertures in a conducting body," Radio Sci., vol. 32, no. 3, pp. 881-898, May/Jun. 1997. [Online]. Available: http://doi.wiley.com/10.1029/96RS03689

[41] P. Liang, Q. Wu, and D. Su, "Characteristic mode analysis of aperture antennas based on duality principle," in Proc. 11th Eur. Conf. Antennas Propag., vol. 3. Mar. 2017, pp. 3004-3008. [Online]. Available: http://ieeexplore.ieee.org/document/7928337/

[42] T. Rozzi and M. Mongiardo, Open Electromagnetic Waveguides. London, U.K.: IET Press, 1997.

[43] E. Anderson et al., LAPACK Users' Guide, 3rd ed. Philadelphia, PA, USA: SIAM, 1999.

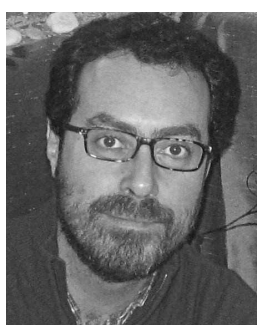

Raúl Rodríguez-Berral was born in Casariche, Seville, Spain, in 1978. He received the Licenciado (M.Sc.) and Doctor (Ph.D.) degrees in physics from the Universidad de Sevilla, Seville, Spain, in 2001 and 2008, respectively.

$\mathrm{He}$ is currently an Associate Professor with the Departamento de Física Aplicada 1, Universidad de Sevilla. His current research interests include the study of the spectrum and the excitation of periodic and nonperiodic planar structures and highfrequency circuit modeling.

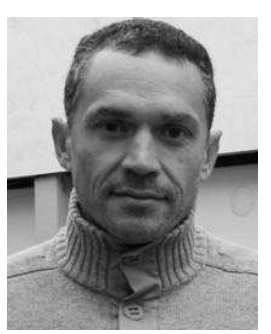

Francisco Mesa (M'93-SM'11-F'14) was born in Cádiz, Spain, in 1965. He received the Licenciado and $\mathrm{Ph} . \mathrm{D}$. degrees in physics from the Universidad de Sevilla, Seville, Spain, in 1989 and 1991, respectively.

He is currently a Professor with the Departamento de Física Aplicada 1, Universidad de Sevilla. His current research interests include electromagnetic propagation/radiation in planar structures.

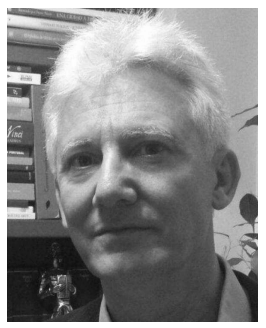

Francisco Medina (M'90-SM'01-F'10) was born in Cádiz, Spain, in 1960. He received the Licenciado and Doctor degrees in physics from the Universidad de Sevilla, Seville, Spain, in 1983 and 1987, respectively.

$\mathrm{He}$ is currently a Professor of electromagnetism with the Departamento of Electrónica y Electromagnetismo, Universidad de Sevilla, where he is also the Head of the Microwaves Group. His current research interests include analytical and numerical methods for planar structures, anisotropic materials, and artificial media modeling. He has published a number of book chapters, journal papers, and conference papers on these topics.

Dr. Medina is a member of the Technical Program Committee of the European Microwave Conference. He is an Associate Editor of the International Journal of Microwave and Wireless Technologies and the International Journal of RF and Microwave Computer-Aided Engineering. 